\title{
Ultraspinning instability of anti-de Sitter black holes
}

\author{
Óscar J. C. Dias, ${ }^{a}$ Pau Figueras, ${ }^{a}$ Ricardo Monteiro, ${ }^{b}$ and Jorge E. Santos ${ }^{c}$ \\ ${ }^{a}$ DAMTP, Centre for Mathematical Sciences, University of Cambridge, \\ Wilberforce Road, Cambridge CB3 0WA, United Kingdom \\ ${ }^{b}$ The Niels Bohr International Academy, The Niels Bohr Institute, \\ Blegdamsvej 17, DK-2100 Copenhagen, Denmark \\ ${ }^{c}$ Department of Physics, UCSB, \\ Santa Barbara, CA 93106, U.S.A. \\ E-mail: o.dias@damtp.cam.ac.uk, p.figueras@damtp.cam.ac.uk, \\ monteiro@nbi.dk, jss55@physics.ucsb.edu
}

ABSTRACT: Myers-Perry black holes with a single spin in $d>5$ have been shown to be unstable if rotating sufficiently rapidly. We extend the numerical analysis which allowed for that result to the asymptotically AdS case. We determine numerically the stationary perturbations that mark the onset of the instabilities for the modes that preserve the rotational symmetries of the background. The parameter space of solutions is thoroughly analysed, and the onset of the instabilities is obtained as a function of the cosmological constant. Each of these perturbations has been conjectured to represent a bifurcation point to a new phase of stationary AdS black holes, and this is consistent with our results.

KeYwords: Black Holes in String Theory, AdS-CFT Correspondence, Black Holes

ARXIV EPRINT: 1011.0996 


\section{Contents}

1 Introduction 1

2 Myers-Perry-AdS black holes 4

2.1 Solution 4

2.2 Thermodynamic zero-modes and the ultraspinning regime 5

$\begin{array}{lll}2.3 & \text { Superradiant instability } & 9\end{array}$

3 Perturbations of MP-AdS black holes $\quad 10$

$\begin{array}{ll}3.1 \text { The Lichnerowicz eigenvalue problem } & 10\end{array}$

$\begin{array}{lll}3.2 & \text { Boundary conditions } & 11\end{array}$

$\begin{array}{lll}3.3 & \text { Imposing the TT gauge conditions and the boundary conditions } & 12\end{array}$

4 Results and discussion $\quad 13$

\section{Introduction}

There has been recent progress in understanding the phase diagram of higher-dimensional asymptotically flat vacuum black holes. The purpose of this work is to extend some of the techniques used to the case of asymptotically anti-de Sitter (AdS) black holes.

Let us first review the asymptotically flat case. While the four-dimensional Kerr black hole is unique and (expected to be) stable, the higher-dimensional picture is much richer [1]. In $d=5$, the discovery of the black ring by Emparan and Reall [2] showed that it can have the same conserved charges as the Myers-Perry (MP) black hole [3]. This non-uniqueness discovery triggered the research in higher dimensional black holes and a variety of explicit rotating solutions have been recently found: black Saturns [4], concentric rings [5, 6], orthogonal rings $[7,8]$, and generalisations thereof. Consider solutions rotating on a single plane which are in thermal equilibrium (i.e. in the case that there are disconnected components of the event horizon, these have the same temperature and angular velocity). One interesting fact is that in the zero temperature limit the new solutions and the MP black hole coincide in the same nakedly singular solution [9-11]. In $d>5$, this limit does not exist, and the MP black hole has an unbounded angular momentum for a given mass. In a certain sense, the singular limit is 'resolved'. However, does the MP black hole still connect to the new solutions? In $d>5$, the black ring and the other solutions have not been constructed exactly, but are expected to exist. In fact, approximate methods for solving the Einstein equations in the ultraspinning limit (large angular momentum with respect to the mass scale) indicate that they do exist [12-15].

The answer seems to be that the MP black hole connects to the other solutions through yet more families of black holes. These families bifurcate from the MP branch at the (stationary) onset of the so called 'ultraspinning instabilities'. The first step in understanding 
this connection was given by Emparan and Myers [16], who argued that the singly-spinning (i.e. rotating on a single plane) MP black hole should be unstable if rotating sufficiently rapidly. The reasoning is that if the angular momentum per unit mass is large enough, MP black holes start behaving like black branes, since their horizons become disk-like along the rotation plane in this ultraspinning limit. But it has been shown by Gregory and Laflamme [17] that black branes are unstable and therefore one concludes that MP black holes should be unstable for a sufficiently large (but finite) angular momentum per unit mass. Emparan and Myers further pointed out that at the onset of such an instability, there should exist a stationary perturbation that preserves the rotational symmetries of the background. This perturbation would then signal the existence of a new family of black holes with spherical horizon topology. This is analogous to what happens in the black brane case: the threshold mode of the Gregory-Laflamme instability is the perturbative signal for a family of non-uniform branes [18, 19]. Ref. [12] conjectured that the new families bifurcating from the MP branch, when continued along the phase diagram, would connect continuously to the black ring branch, the black Saturn branch, and so on. Therefore, the connection of non-uniqueness with instabilities provides a partial understanding of the proliferation of higher dimensional black hole phases.

Recently, these conjectures were put on a firmer footing when it was shown numerically that the singly-spinning MP black hole does indeed possess such threshold modes [20, 21]. Furthermore, these perturbations exhibit an underlying harmonic structure and induce deformations on the shape of the horizon which are consistent with the proposal that the new families will connect to the black ring, to the black Saturn, etc. Ref. [20] also conjectured that these classical instabilities can only occur when the black hole possesses at least two distinct local thermodynamic instabilities, as we shall review later. This is a necessary but not sufficient condition. The reason is that local thermodynamic instabilities are associated to the lowest harmonics, and thus with the asymptotic charges, the mass and the angular momentum. Higher harmonics, which cannot change the asymptotic charges and are associated to the bifurcation to new families, should only become unstable for rotations higher than the thermodynamic modes. This conjecture allows for any number of independent angular momenta, and has already been verified in the instability of $d>5$ cohomogeneity-1 MP solutions (equal independent spins in odd $d$ ) [22]. This sector of MP black holes has a regular extremal limit and the angular momentum is bounded from above, yet the conjecture of ref. [20] indicates that an instability is possible. Indeed linear perturbations growing exponentially with time were found, which is an important check since the previous work could only determine the stationary threshold modes.

In this paper, we will extend the results in refs. [20, 21] to the asymptotically AdS case. The properties of asymptotically AdS spacetimes have been greatly explored due to the AdS/CFT correspondence, which equates quantum gravity (string theory) in an asymptotically AdS spacetime to a conformal field theory (CFT) living on the boundary of that spacetime $[23,24]$. In particular, the phase diagram of black holes in AdS is in direct correspondence to the phases of the dual CFT at finite temperature. Therefore, even if many higher-dimensional black hole solutions present classical instabilities, or do not dominate thermodinamically the gravitational partition function, they still provide a valuable insight into the CFT phases. 


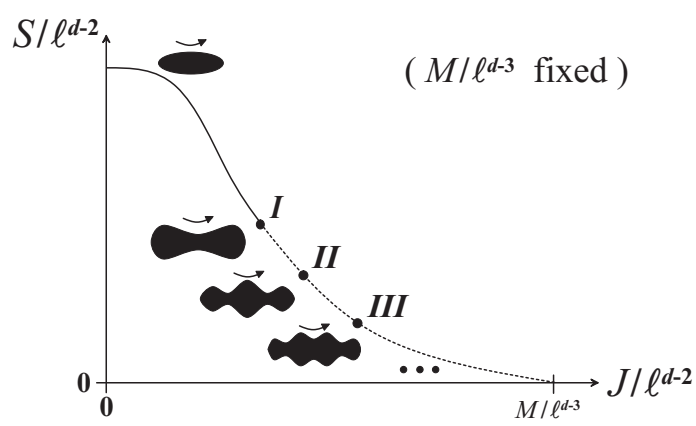

Figure 1. Phase diagram of singly-spinning MP-AdS black holes in $d \geq 6$. We plot the entropy $S$ vs. the angular momentum $J$, at a fixed value of the mass $M$, in units of the AdS curvature radius $\ell$. The figure illustrates the conjecture of ref. [25]. At sufficiently large spin the MP-AdS solution becomes unstable for axisymmetric perturbations (dashed line), and at the threshold of the instability a new branch of black holes with a central pinch appear $(I)$. As the spin grows, new branches of black holes with further axisymmetric pinches $(I I, I I I, \ldots)$ appear. We determine numerically the points where the new branches appear, but it is not yet known in which directions they run.

Black rings and black Saturns in AdS have been constructed in certain approximations [25], ${ }^{1}$ and the conjectures of ref. [12] regarding the connections between singlyspinning black hole families were extended to the asymptotically AdS case also in ref. [25]. Notice that singly-spinning MP-AdS black holes [27] (and indeed any asymptotically AdS stationary black hole [28]) present a BPS-type upper bound on their angular momentum, $|J|<M \ell$ (with $M$ being the mass and $\ell$ the cosmological length), while there is no such bound in the asymptotically flat case. However, the instabilities/bifurcations are still expected as we increase the rotation up to that bound [25] (see figure 1).

In this paper we will numerically determine the dependence of the stationary threshold modes on the cosmological constant. The procedure is an extension to $d \geq 6$ of the $d=4$ Kerr-AdS analysis [29], where no classical instability was found; the latter was itself an extension of the Schwarzschild-AdS case [30].

We confirm the conjecture of ref. [20], which gives a necessary but not sufficient condition for the onset of the ultraspinning instability. In this paper we generalise the previous formulation so that it can be applied to the asymptotically AdS case. We find that all bifurcations occur when the MP-AdS black hole is unstable under superradiance [31-33], which implies that the new families of black holes will inherit this instability.

Before proceeding, let us mention that asymptotically flat singly-spinning MP black holes also suffer from an instability that breaks their axisymmetry [34, 35]. This occurs even in $d=5$, and for $d \geq 6$ it sets in for lower rotations than the ultraspinning instability that we study here. However, the threshold mode is not associated to new stationary black holes. It would be interesting to find whether this non-axisymmetric instability also

\footnotetext{
${ }^{1}$ See ref. [26] for the extension of the more systematic blackfold approach of refs. [13-15] to asymptotically AdS black holes.
} 
extends to asymptotically AdS black holes and to understand how it relates to the AdS superradiant instability.

This paper is organised as follows. In section 2, we review the properties of singlyspinning MP-AdS black holes and discuss the ultraspinning instability. In section 3, we detail the linear perturbations problem that is solved numerically. We conclude in section 4 , with the discussion of the results.

\section{Myers-Perry-AdS black holes}

In this section, we will start by reviewing the properties of singly-spinning MP-AdS black holes. Although for such solution the angular momentum (at fixed mass) has an upper bound, we provide a thermodynamic argument, discussed in subsection 2.2 , according to which these black holes may be afflicted by the ultraspinning instability. This motivates the numerical work, described in later sections, of searching for the onset of the instability. We conclude this section by noting that all the AdS black holes potentially afflicted from the ultraspinning instability are also afflicted by the AdS superradiant instability.

\subsection{Solution}

The four-dimensional Kerr-AdS black hole was found by Carter [36], and its extension to higher dimensions, when rotation on a single plane is considered, was obtained by Hawking, Hunter and Taylor [27]. ${ }^{2}$ The metric is given by

$$
\begin{aligned}
d s^{2}= & -\frac{\Delta_{r}}{\Sigma}\left(d t-\frac{a \sin ^{2} \theta}{\Xi} d \phi\right)^{2}+\frac{\sin ^{2} \theta \Delta_{\theta}}{\Sigma}\left[\frac{r^{2}+a^{2}}{\Xi} d \phi-a d t\right]^{2}+\frac{\Sigma}{\Delta_{r}} d r^{2}+\frac{\Sigma}{\Delta_{\theta}} d \theta^{2} \\
& +r^{2} \cos ^{2} \theta d \Omega_{(d-4)}^{2},
\end{aligned}
$$

where $d \Omega_{(d-4)}^{2}$ is the line element of a unit-radius $(d-4)$-sphere and

$\Delta_{r}=\left(r^{2}+a^{2}\right)\left(1+\frac{r^{2}}{\ell^{2}}\right)-\frac{r_{m}^{d-3}}{r^{d-5}}, \quad \Delta_{\theta}=r^{2}+a^{2} \cos ^{2} \theta, \quad \Sigma=r^{2}+a^{2} \cos ^{2} \theta, \quad \Xi=1-\frac{a^{2}}{\ell^{2}}$.

This solution of the Einstein equations with negative cosmological constant, $R_{\mu \nu}=-(d-$ 1) $\ell^{-2} g_{\mu \nu}$, is parameterised by three length scales, namely the AdS curvature radius $\ell$, the mass-radius $r_{m}$ and the rotation parameter $a$. The Komar mass $M$ and angular momentum $J$ of the black hole are given in terms of these parameters by [37]

$$
M=\frac{\mathcal{A}_{d-2} r_{m}^{d-3}}{8 \pi G \Xi^{2}}\left(1+\frac{d-4}{2} \Xi\right), \quad J=\frac{\mathcal{A}_{d-2}}{8 \pi G} \frac{r_{m}^{d-3}}{\Xi^{2}} a,
$$

where $\mathcal{A}_{d-2}=2 \pi^{(d-1) / 2} / \Gamma[(d-1) / 2]$ is the volume of a unit-radius $(d-2)$-sphere, and $G$ denotes Newton's constant which we set to one $(G=1)$. The solution satisfies the BPS-like bound [28]

$$
|J|<M \ell \quad \Leftrightarrow \quad|a|<\ell .
$$

\footnotetext{
${ }^{2}$ Since the Hawking-Hunter-Taylor solution usually refers to the five-dimensional black hole with two independent spins, also found in [27], we will refer to the black hole represented by (2.1) simply as (singlyspinning) Myers-Perry-AdS black hole.
} 
The metric (2.1) does not describe a black hole if this bound is saturated. In the limit $|a| \rightarrow$ $\ell$ either the charges (mass and angular momentum) and the horizon equator circumference of the black hole diverge, or the horizon vanishes and the solution is nakedly singular.

The event horizon lies at the largest real root $r=r_{+}$of $\Delta_{r}(r)=0$. The horizon angular velocity measured with respect to a non-rotating frame at infinity is $[37,38]$

$$
\Omega_{H}=\frac{a}{r_{+}^{2}+a^{2}}\left(1+\frac{r_{+}^{2}}{\ell^{2}}\right)
$$

while the temperature and entropy of the black hole are [37]

$$
\begin{aligned}
T_{H} & =\frac{1}{4 \pi r_{+}\left(a^{2}+r_{+}^{2}\right)}\left(r_{+}^{2}\left[d-3+(d-1) \frac{r_{+}^{2}}{\ell^{2}}\right]+a^{2}\left[d-5+(d-3) \frac{r_{+}^{2}}{\ell^{2}}\right]\right), \\
S & =\frac{\mathcal{A}_{d-2}}{4} \frac{\left(r_{+}^{2}+a^{2}\right) r_{+}^{d-4}}{\Xi}
\end{aligned}
$$

In $d=4$, the temperature vanishes for $|a|=r_{+} \sqrt{\left(3 r_{+}^{2}+\ell^{2}\right)\left(\ell^{2}-r_{+}^{2}\right)^{-1}}$. However, as a consequence of the constraint (2.3), we have an extremal regular (i.e. with finite size horizon area) black hole if and only if $r_{+} / \ell<3^{-1 / 2}$. In $d=5$, like in the asymptotically flat space limit $\ell \rightarrow \infty$, the temperature goes to zero at the singular limit $|a| \rightarrow r_{m}$, whereas the bound on the rotation parameter for a finite $r_{+}$is (2.3). For $d \geq 6$, the temperature can never vanish and thus there is no extremal solution. The parameter space is bounded only by (2.3). This is in sharp contrast with the asymptotically flat case, in which MP black holes rotating on a single plane in $d \geq 6$ have no upper bound on their angular momentum.

\subsection{Thermodynamic zero-modes and the ultraspinning regime}

The condition of local thermodynamic stability for a black hole with charges $M, J_{i}$ and entropy $S$ is the positivity of the Hessian

$$
-S_{\alpha \beta} \equiv-\frac{\partial^{2} S\left(x_{\gamma}\right)}{\partial x_{\alpha} \partial x_{\beta}}, \quad x_{\alpha}=\left(M, J_{i}\right) .
$$

We write the condition in its most general form, i.e. taking into account the several angular momenta allowed in higher dimensions, $i=1, \ldots,\lfloor(d-1) / 2\rfloor$ (where $\lfloor$.$\rfloor stands for$ the smallest integer part). In this section, we will review the arguments of refs. $[20,22]$ which relate the eigenvalues of this thermodynamic Hessian to the occurrence of classical instabilities, namely the ultraspinning instability.

There are two general properties of this Hessian for AdS black holes: (i) For all (nonextremal) asymptotically flat vacuum black holes, in any $d$, the Hessian has at least one negative eigenvalue [22]; so, by continuity, black holes whose size is much smaller than the AdS curvature radius should be thermodynamically unstable. (ii) Large black holes in AdS, however, are expected to be thermodynamically stable, and thus the Hessian should be positive definite.

Let us take the $d=4$ Kerr-AdS case, where we have a $2 \times 2$ thermodynamic Hessian. One of the eigenvalues is positive in the entire parameter space. However, the second 


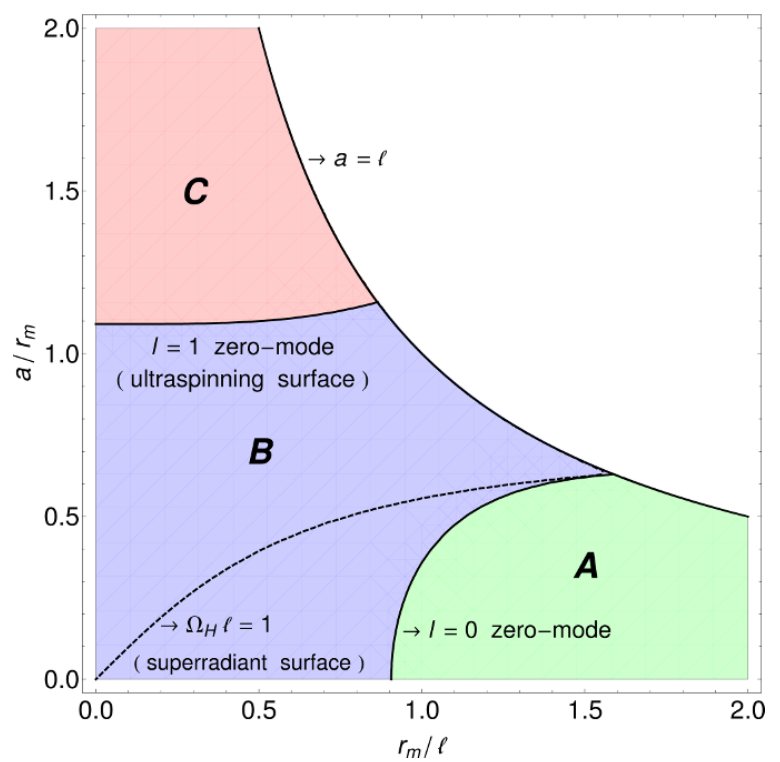

Figure 2. Parameter space of singly-spinning Myers-Perry-AdS black holes in $d=6$ : dimensionless rotation parameter $a / r_{m}$ as a function of the dimensionless mass-radius parameter $r_{m} / \ell$. Regular black holes exist only for $a<\ell$. The thermodynamic $l=0$ and $l=1$ zero-mode curves, described by (2.7) and (2.8), are plotted. The thermodynamic $l=1$ zero-mode curve defines also the ultraspinning surface, above which (region $C$ ) the black holes might be ultraspinning unstable (later section 4 and figure 4 confirm this is indeed the case). The superradiant curve $\Omega_{H} \ell=1$ is also plotted. Above this curve all black holes are superradiant unstable. For $d>6$ the plot is qualitatively very similar.

eigenvalue can be negative, null or positive depending on the parameters. The null point is where the specific heat at constant angular velocity diverges, changing sign. We shall say that, at the surface in parameter space where the Hessian becomes degenerate, we have a thermodynamic zero-mode, corresponding to the eigenvector with zero eigenvalue. The situation is analogous in the singly-spinning $d=5$ case (say $J_{2}=0$ ), with two eigenvalues being always positive, and one changing sign. In the $d \geq 6$ singly-spinning case (say $J_{i}=0$ for $i>1$ ) things are more interesting. Two of the eigenvalues of the thermodynamic Hessian change sign, and all the others remain positive throughout the parameter space. So there will be two thermodynamic zero-modes, marking the onsets of two distinct local thermodynamic instabilities.

Let us consider the parameterisation $\left(r_{+}, a\right)$, which uniquely specifies a singly-spinning MP-AdS black hole, for a given AdS curvature radius $\ell$. Recall that $|a|<\ell$ is the bound on the parameter space, as represented in figure 2 for $d=6$ (it is qualitatively similar in $d>6)$. Now, for large $r_{+} / \ell$, the black hole is thermodynamically stable. As we decrease $r_{+}$, one of the eigenvalues changes sign first, which corresponds to the transition between regions $A$ and $B$ in figure 2. Let us label the associated zero-mode as the $l=0$ zero-mode, 
for reasons that will become apparent later. Its parameter space locus is: ${ }^{3}$

Thermodynamic $l=0$ zero-mode $(d \geq 4): \quad r_{+}^{2}=\frac{d-3}{2(d-1)}\left(a^{2}+\ell^{2}+\sqrt{a^{4}-\gamma_{d} a^{2} \ell^{2}+\ell^{4}}\right)$, where $\gamma_{d} \equiv 2\left(d^{2}-6 d+1\right) /(d-3)^{2}$.

The $l=0$ zero-mode is present for any $d \geq 4$. However, for $d \geq 6$ there is an additional zero-mode, corresponding to the second eigenvalue that becomes negative as we lower $r_{+}$ for fixed $a$. This corresponds to the transition between regions $B$ and $C$ in figure 2. Let us label the associated zero-mode with $l=1$. Its parameter space locus is:

Thermodynamic $l=1$ zero-mode $(d \geq 6): \quad r_{+}^{2}=\frac{d-3}{2(d-1)}\left(a^{2}+\ell^{2}-\sqrt{a^{4}-\gamma_{d} a^{2} \ell^{2}+\ell^{4}}\right)$.

What happens as we decrease $r_{+}$further for fixed $a$ ? As discussed in the Introduction, singly-spinning MP black holes were conjectured to be unstable if rotating too rapidly, i.e. $|a| \gg r_{+}$, since they start behaving like black branes [16]. In the asymptotically AdS case, there should also be an analogous unstable regime [25]. Now, there can be two types of instabilities. One for which the perturbations break the rotational symmetry of the background, i.e. the $\partial_{\phi}$ Killing vector of $(2.1)$; see $[34,35]$ for the analysis of these perturbations in the asymptotically flat case. And one for which the spatial isometries of the background are preserved. Instabilities of the latter type admit stationary threshold modes, which may correspond to bifurcations to new black hole phases [12, 16]. Ref. [16] suggested that an order of magnitude estimate for the critical rotation at the bifurcation point could be given by what we described above as the $l=1$ zero mode. In fact, this point marks the transition to the black brane-like behaviour and it only occurs for $d \geq 6$. The subsequent understanding of the problem allowed for a more precise formulation of the conjecture, which can also be extended beyond the singly-spinning case.

Indeed, the ultraspinning conjecture of ref. [20] proposes that instabilities whose onset is a stationary and axisymmetric mode, i.e. a mode which gives a bifurcation to a new stationary black hole family, can only occur after the $l=0$ and $l=1$ zero-modes, i.e. in region $C$ of figure 2 in the present case. This is a necessary but not sufficient condition. The reasoning is that the onset of these instabilities corresponds to the $l \geq 2$ zero-modes, as we shall review below.

Ref. [40], in the static case, and refs. [22, 41], in the general case, showed that a zeromode (or a negative mode, the eigenvector of a negative eigenvalue) of the thermodynamic Hessian (2.6) is also a stationary and axisymmetric zero-mode (negative mode) of the black hole Euclidean action. Since we are dealing with stationary and axisymmetric modes, this result extends to the Lorentzian action. In the case of a zero-mode, it corresponds to a classical perturbation of the black hole which preserves its temperature and angular velocities, but changes its asymptotic charges (mass and angular momenta). The change

\footnotetext{
${ }^{3}$ Notice that the Hawking-Page transition [39] occurs for $r_{+} / \ell$ larger than (2.7). This implies that hot AdS space is preferred (in the grand-canonical ensemble) over these black holes if $r_{+}<\ell$, in any $d$. Global thermodynamic stability is stricter than local thermodynamic stability.
} 
in the asymptotic charges can simply be inferred from the eigenvector $\left(\delta M, \delta J_{i}\right)$ of the Hessian (2.6) associated with the null eigenvalue. In the case of a negative mode, it corresponds to both: (i) an off-shell perturbation of the black hole which makes the (Euclidean) gravitational partition function pathological, the expected signal of a local thermodynamic instability; and (ii) an on-shell stationary perturbation of a black brane, where the negative eigenvalue corresponds to (minus) the 'mass-squared' coming from the dimensional reduction along the brane directions. This connection is the basis of the Gubser-Mitra conjecture [42, 43].

The first example of the relation between negative modes of the action and thermodynamic stability was the determination of the negative mode of the Schwarzschild black hole [44]. The existence of such mode is a direct consequence of the fact the Schwarzschild black hole has negative specific heat. Indeed, the extension to the Schwarzschild-AdS case [30] showed that the relevant mode changes sign exactly where the specific heat changes sign, as expected. This result was extended to the Kerr-AdS case [29], where the numerical techniques used in $[20-22,45]$, and also in this paper, were applied first to a black hole stability problem.

Now we can justify the labels $l=0$ and $l=1$ used before, following [20]. They are based on the harmonic structure of the metric perturbations corresponding to the zeromodes. The $l=0$ zero-mode has no nodes, i.e. the metric perturbation does not vanish anywhere on the horizon, whereas the $l=1$ zero-mode has one node, as plotted in [20] for the singly-spinning MP case. Now, the $l=0$ and $l=1$ zero-modes are associated with the asymptotic charges of the spacetime. ${ }^{4}$ This is consistent with the fact that they are determined from the thermodynamic Hessian (2.6), the changes in the charges being proportional to the eigenvector $\left(\delta M, \delta J_{i}\right)$. Notice that, being signalled by the Hessian of $S\left(M, J_{i}\right)$, i.e. by the equation of state of the background black hole family, the $l=0$ and $l=1$ zero-modes can only change the black hole into another black hole of the same family, e.g. another MP black hole. ${ }^{5}$

What is found by solving the problem of axisymmetric perturbations is that instabilities appear with new stationary zero-modes, not predicted by the thermodynamic Hessian. We refer to them as non-thermodynamic zero-modes. Their harmonic structure - the number of nodes, found to be $l \geq 2$ - is consistent with the deformations of the event horizon proposed in refs. [12, 25]; see figure 1. These higher harmonics cannot change the asymptotic charges, as exemplified explicitly in [22]. The perturbed black holes will have the same mass and angular momenta as the unperturbed solutions, and also, as imposed by

\footnotetext{
${ }^{4}$ Ref. [22] analysed MP black holes with equal spins in odd $d$, which have the convenient property of being cohomogeneity-1. In that case, the number $l$ labels a precise harmonic of the $C P^{N}$ base space, and it can be shown that the $l=0$ harmonic is associated to the mass only (indeed $\delta J_{i}=0$ in the Hessian eigenvector), while the $l=1$ harmonic is associated to the angular momenta only (indeed $\delta M=0$ in the Hessian eigenvector). In the singly-spinning black hole both the $l=0$ and $l=1$ 'harmonics' change the mass and the angular momentum.

${ }^{5}$ In the present paper, these zero-modes give another singly-spinning MP-AdS black hole. However, in the cohomogeneity-1 MP(-AdS) case [22], the $l=1$ zero-mode breaks the symmetries between the spins, as predicted by the eigenvector $\left(\delta M, \delta J_{i}\right)$ having distinct $\delta J_{i}$. The zero-mode therefore takes the black hole out of the equal spins sector, but still in the general MP(-AdS) family.
} 
the boundary conditions, the same temperature and angular velocities. Therefore, either they correspond to trivial gauge modes or to bifurcations to new black hole solutions. We have shown in specific cases $[21,22]$ that no regular gauge modes are allowed. We conclude that the $l \geq 2$ zero-modes mark a black hole bifurcation, and also the onset of a classical ultraspinning instability, one per each mode. That these linear modes do grow exponentially with time was verified in [22].

The ultraspinning conjecture of ref. [20] predicts that zero-modes with $l \geq 2$ can only occur for rotations higher than that of the $l=1$ zero-mode. In the case of singly-spinning MP-AdS black holes, this corresponds to the region $C$ of figure 2. This is based on the examples known, and on the intuition that higher harmonics should be more stable than lower harmonics. In general, when several distinct angular momenta are present, several different zero-modes with $l=1$ may exist, as exemplified in [22]. So the ultraspinning surface (in our case, the surface between $B$ and $C$ ) is defined as the surface in parameter space which encloses the region where the thermodynamic Hessian (2.6) has less than two negative eigenvalues (in our case, regions $A$ and $B$ ).

Since this thermodynamic criterion is a necessary but not sufficient condition, to confirm the presence of these instabilities we still have to solve the linear perturbations problem, and look for the $l \geq 2$ non-thermodynamic zero-modes. This study is done in section 3 .

\subsection{Superradiant instability}

Before studying the ultraspinning instability of the singly-spinning MP-AdS black hole, we recall that this is not the only instability present in the system. Indeed, it competes with the well-known superradiant instability that afflicts rotating AdS black holes whenever $\Omega_{H} \ell>1$ [31-33]. The superradiant instability is associated to perturbations that break the symmetry generated by $\partial_{\phi}$, i.e. it requires perturbations whose angular momentum quantum number $m$ along the $\phi$-direction is non-vanishing. The mechanism of the instability is simple. A gravitational wave co-rotating with the background black hole can extract rotational energy from the black hole if its frequency $\omega$ satisfies the relation $\omega<m \Omega_{H}$, where $\Omega_{H}$ is the angular velocity of the black hole. This phenomenon is known as superradiance. On the other hand, the AdS gravitational potential effectively acts as an asymptotically reflecting box. The multiple superradiant amplifications and reflections drive the system unstable if and only if $\Omega_{H} \ell>1$.

Since there is a competition between the two instabilities, it is relevant to ask whether the ultraspinning instability occurs when $\Omega_{H} \ell \leq 1$. We find a negative answer. Indeed, in figure 2 we represent the superradiant surface, $\Omega_{H} \ell=1$, by a dashed line. It lays entirely in region $B$. Black holes above this surface have $\Omega_{H} \ell>1$, which includes all the black holes in region $C$ that might also be afflicted by the ultraspinning instability. Therefore, all the AdS black holes that suffer from the ultraspinning instability that we identify in the next section are also likely to be unstable under superradiance. 


\section{Perturbations of MP-AdS black holes}

In this section, we will detail the linear perturbation problem, which consists in studying the spectrum of the Lichnerowicz operator on the black hole background. We will list the equations to be solved and the boundary conditions, and we will briefly discuss their numerical implementation.

\subsection{The Lichnerowicz eigenvalue problem}

To find the onset of the ultraspinning instability we have to solve the linearised Einstein equations with a negative cosmological constant. We search for stationary modes that depend on the radial and polar coordinates, $r$ and $\theta$, but preserve the $\mathbb{R} \times \mathrm{U}(1) \times \mathrm{SO}(d-$ 3) symmetries of the background MP-AdS black hole (2.1). The general ansatz for the perturbed metric $h_{\mu \nu}$ satisfying these conditions is: ${ }^{6}$

$$
\begin{aligned}
d s^{2}= & -\frac{\Delta_{r}(r)}{\Sigma(r, \theta)} e^{\delta \nu_{0}}\left[d t-\frac{a \sin ^{2} \theta}{\Xi} e^{\delta \omega} d \phi\right]^{2}+\frac{\sin ^{2} \theta \Delta_{\theta}(\theta)}{\Sigma(r, \theta)} e^{\delta \nu_{1}}\left[\frac{r^{2}+a^{2}}{\Xi} d \phi-a e^{-\delta \omega} d t\right]^{2} \\
& +\frac{\Sigma(r, \theta)}{\Delta_{r}(r)} e^{\delta \mu_{0}}[d r-\delta \chi \sin \theta d \theta]^{2}+\frac{\Sigma(r, \theta)}{\Delta_{\theta}(\theta)} e^{\delta \mu_{1}} d \theta^{2}+r^{2} \cos ^{2} \theta e^{\delta \Phi} d \Omega_{(d-4)}^{2},
\end{aligned}
$$

where $\left\{\delta \nu_{0}, \delta \nu_{1}, \delta \mu_{0}, \delta \mu_{1}, \delta \omega, \delta \chi, \delta \Phi\right\}$ are small quantities that describe our perturbations, and are functions of $(r, \theta)$ only. We will solve numerically the coupled partial differential equations (PDEs) that govern these perturbations. We choose to work in the tracelesstransverse (TT) gauge,

$$
h^{\mu}{ }_{\mu}=0 \quad \text { and } \quad \nabla^{\mu} h_{\mu \nu}=0 .
$$

The linearised Einstein-AdS equations are:

$$
\left(\tilde{\Delta}_{L} h\right)_{\mu \nu} \equiv-\nabla_{\rho} \nabla^{\rho} h_{\mu \nu}-2 R_{\mu}^{\rho}{ }_{\nu}^{\sigma} h_{\rho \sigma}=0,
$$

where the operator $\tilde{\Delta}_{L}$ relates to the standard Lichnerowicz operator $\Delta_{L}$ as

$$
\left(\tilde{\Delta}_{L} h\right)_{\mu \nu}=\left(\Delta_{L} h\right)_{\mu \nu}+2(d-1) \ell^{-2} h_{\mu \nu} .
$$

Following the strategy used to study the ultraspinning instability in asymptotically flat black holes in [20-22], we will actually consider a more general eigenvalue problem,

$$
\left(\tilde{\Delta}_{L} h\right)_{\mu \nu}=\lambda h_{\mu \nu} .
$$

More concretely, we will be looking for negative modes $(\lambda<0)$ of the operator $\tilde{\Delta}_{L}$.

This eigenvalue problem arises in two instances. One is the computation of quadratic quantum corrections to the gravitational partition function in the saddle point approximation [44] (see [29] for the application to the Kerr-AdS black hole). The quantum corrections

\footnotetext{
${ }^{6}$ Stationarity and axisymmetry lead to a metric perturbation independent of $t$ and $\phi$, respectively. The transverse $S^{d-4}$ line element is left unperturbed, apart from its $(r, \theta)$-dependent scale factor, because we wish to preserve the $\mathrm{SO}(d-3)$ isometry. On the other hand, the components $t r, t \theta, \phi r, \phi \theta$ can be discarded: the corresponding TT gauge conditions and the linearised equations of motion can be solved by separation of variables in $r$ and $\theta$, and regularity implies that those components vanish.
} 
present a pathology whenever there is a negative mode. As we have mentioned, each negative eigenvalue of the thermodynamic Hessian (2.6) gives a negative mode, which we refer to as being thermodynamic.

In the asymptotically flat case, (3.5) also governs the gravitational perturbations of the form $e^{\mathrm{i} \sqrt{-\lambda} z} h_{\mu \nu}$ of the rotating black string that is constructed by adding a flat extra dimension $z$ to the MP geometry. The AdS analogue are the warped AdS black strings constructed in ref. [46]. These solutions are the generalisations of the standard warped AdS black string, whose stability was studied in [47], in the case where the transverse black hole is also asymptotically AdS. ${ }^{7}$

Our strategy (following refs. $[20,29])$ to study the classical perturbations $(\lambda=0)$ of the black hole is to look for a solution of (3.5), i.e. a negative mode of the black hole, and then vary the rotation parameter $a$ of the black hole until the negative mode becomes a zeromode, i.e. until $\lambda \rightarrow 0$. This strategy is motivated by the availability of powerful numerical methods for solving eigenvalue equations of the form (3.5). This strategy is motivated by the availability of powerful numerical methods for solving eigenvalue equations of the form (3.5). In particular, after an adequate discretisation scheme using spectral methods [48], (3.5) reduces to an algebraic eigenvalue problem which we then solve using the inbuilt routine Eingenvalues of Mathematica.

\subsection{Boundary conditions}

To solve the Lichnerowicz eigenvalue system of equations (3.5) we have to impose boundary conditions on the metric perturbations (3.1). More concretely, we have to specify boundary conditions at the horizon, $r=r_{+}$, at the asymptotic infinity, $r \rightarrow \infty$, at the rotation axis $\theta=0$, and at the equator $\theta=\pi / 2$. The strategy to find the appropriate boundary conditions was already discussed in great detail in section 4 of ref. [21], where the ultraspinning instability in asymptotically flat MP black holes was studied. The discussion there translates straightforwardly to the present situation, and hence we shall be brief.

We impose regularity of the metric perturbations on the event horizon by demanding regularity of the Euclideanised perturbed geometry. The idea is to peform the standard Euclidean continuation of the black hole metric, and find the conditions required to have regularity at the bolt (Euclideanised horizon). For the background black hole, this demands that we identify $(\tau, \phi) \sim(\tau, \phi+2 \pi) \sim\left(\tau+\beta, \phi-\mathrm{i} \Omega_{H} \beta\right)$, where $\tau=\mathrm{i} t$ is the Euclidean time, $\beta=1 / T_{H}$ is the inverse of the black hole temperature (2.5), and $\Omega_{H}$ is its angular velocity (2.4). The boundary conditions for the metric perturbations $h_{\mu \nu}$ can now be determined by demanding that $h_{\mu \nu} d x^{\mu} d x^{\nu}$ is a regular symmetric 2-tensor on the background manifold. This requires the following boundary conditions at the horizon, located at $\rho \equiv\left[4\left(r-r_{+}\right) / \Delta_{r}^{\prime}\left(r_{+}\right)\right]^{1 / 2}=0$,

$$
\delta \chi, \delta \omega=O\left(\rho^{2}\right), \quad \delta \nu_{0}-\delta \mu_{0}=O\left(\rho^{2}\right), \quad \partial_{\rho} \delta \mu_{1}, \partial_{\rho} \delta \nu_{1}, \partial_{\rho} \delta \Phi=O(\rho) .
$$

Note that we have not just imposed regularity of the perturbed metric. If $h_{\mu \nu} d x^{\mu} d x^{\nu}$ is a regular 2-tensor, then perturbations obeying (3.6) preserve the angular velocity and

\footnotetext{
${ }^{7}$ These should not be confused with the uniform black string solutions of ref. [57], whose stability problem is not given by eq. (3.5).
} 
temperature of the background black hole. This is a fundamental property to make the connection between the classical ultraspinning instability and black hole thermodynamics that was discussed in section 2 [22].

To find the boundary conditions that the metric perturbations must satisfy at the axis of rotation $(\theta=0)$, where $\partial_{\phi}$ vanishes, we proceed in the same way and require that $h_{\mu \nu} d x^{\mu} d x^{\nu}$ is a regular symmetric 2 -tensor, i.e. the components $h_{\mu \nu}$ are regular when expressed in coordinates where the background metric components are regular. Regularity as $\theta \rightarrow 0$ demands:

$$
\delta \nu_{1}-\delta \mu_{1}=O\left(\theta^{2}\right), \quad \partial_{\theta} \delta \chi, \partial_{\theta} \delta \omega, \partial_{\theta} \delta \mu_{0}, \partial_{\theta} \delta \nu_{0}, \partial_{\theta} \delta \Phi=O(\theta)
$$

Analogously, at the equator, $\theta=\pi / 2$, we impose the boundary conditions:

$$
\delta \chi=O(x), \quad \delta \Phi-\delta \mu_{1}=O\left(x^{2}\right), \quad \partial_{x} \delta \omega, \partial_{x} \delta \mu_{0}, \partial_{x} \delta \nu_{0}, \partial_{x} \delta \nu_{1}=O(x),
$$

as $x=\cos \theta \rightarrow 0$.

We have explicitly checked that the boundary conditions (3.6), (3.7) and (3.8) are consistent both with the Lichnerowicz eigenvalue equations (3.5) and the TT gauge conditions (3.2). Indeed, the first term in the series expansion of the eigenvalue equations vanishes after we impose (3.6), (3.7) and (3.8). On the other hand, we can use the TT gauge conditions to express, e.g. $\left\{\delta \nu_{0}, \delta \nu_{1}, \delta \Phi\right\}$ as functions of $\left\{\delta \mu_{0}, \delta \mu_{1}, \delta \omega, \delta \chi\right\}$ and their first derivatives. Again, the first term of a series expansion of these TT gauge conditions is consistent with (3.6), (3.7) and (3.8).

At spatial infinity, $r \rightarrow \infty$, our second order equations of motion allow for two radial dependences of the metric perturbation. We require that the boundary conditions preserve the asymptotics of the background spacetime, in the sense that not only (in our BoyerLindquist coordinates)

$$
\left.h_{\mu \nu}\right|_{r \rightarrow \infty} \sim \frac{1}{r^{\alpha}} \rightarrow 0
$$

for some constant $\alpha>0$ that depends on the particular metric component and the number of spacetime dimensions $d$, but also in the sense that the perturbations are normalisable. The negative modes are "gravitons with a positive mass", and normalisability requires that we pick the fastest decaying mode (cf. the non-rotating case [30]). This happens to be the mode that our numerical code can identify straightforwardly.

\subsection{Imposing the TT gauge conditions and the boundary conditions}

We wish to solve the Lichnerowicz eigenvalue problem (3.5) for the seven metric perturbations described in (3.1), namely $\left\{\delta \mu_{0}, \delta \mu_{1}, \delta \chi, \delta \omega, \delta \nu_{0}, \delta \nu_{1}, \delta \Phi\right\}$, subject to the TT gauge conditions (3.2). The latter eliminate three functions. We choose to solve the gauge conditions (3.2) for $\left\{\delta \nu_{0}, \delta \nu_{1}, \delta \Phi\right\}$ in terms of $\left\{\delta \mu_{0}, \delta \mu_{1}, \delta \chi, \delta \omega\right\}$ and their first derivatives. Plugging this information in the full set of the perturbation equations (3.5), we find that only 
four equations remain of second order in $\left\{\delta \mu_{0}, \delta \mu_{1}, \delta \chi, \delta \omega\right\}$. Explicitly, these equations are:

$$
\begin{aligned}
\left(\tilde{\triangle}_{L} h\right)_{r r} & =\lambda h_{r r}, \\
\left(\tilde{\triangle}_{L} h\right)_{r \theta} & =\lambda h_{r \theta}, \\
\left(\tilde{\triangle}_{L} h\right)_{\theta \theta} & =\lambda h_{\theta \theta}, \\
a\left(\tilde{\triangle}_{L} h\right)_{t t} & +\frac{\Xi\left(r^{2}+a^{2}+a^{2} \sin ^{2} \theta\right)}{\left(r^{2}+a^{2}\right) \sin ^{2} \theta}\left(\tilde{\triangle}_{L} h\right)_{t \phi}+\frac{a \Xi^{2}}{\left(r^{2}+a^{2}\right) \sin ^{2} \theta}\left(\tilde{\triangle}_{L} h\right)_{\phi \phi} \\
& =\lambda\left[a h_{t t}+\frac{\Xi\left(r^{2}+a^{2}+a^{2} \sin ^{2} \theta\right)}{\left(r^{2}+a^{2}\right) \sin ^{2} \theta} h_{t \phi}+\frac{a \Xi^{2}}{\left(r^{2}+a^{2}\right) \sin ^{2} \theta} h_{\phi \phi}\right],
\end{aligned}
$$

and they describe the final set of equations that we have to solve. A non-trivial consistency check of our procedure is to verify that the final equations (3.10) imply that the remaining equations in (3.5) are automatically satisfied (the latter equations are of third order in the independent perturbation functions once the TT gauge conditions have been imposed). We have explicitly verified that this is the case.

We will solve numerically the final eigenvalue problem (3.10) using spectral methods (see e.g. [48]). To do so, we find it convenient to introduce new radial and polar coordinates,

$$
y=\frac{r}{r_{+}}-1, \quad x=\cos \theta,
$$

such that $0 \leq y \leq \infty$ and $0 \leq x \leq 1$. The implementation of the method is simpler if all functions obey Dirichlet boundary conditions on all the boundaries. Therefore, we redefine our independent functions according to:

$$
\begin{array}{rlrl}
q_{1}(y, x) & =\left(\frac{r}{r_{+}}-1\right) x(1-x) \delta \mu_{0}(y, x), & & q_{2}(y, x)=r_{m}^{-1}(1-x) \delta \chi(y, x), \\
q_{3}(y, x)=\left(\frac{r}{r_{+}}-1\right) x(1-x) \delta \mu_{1}(y, x), & q_{4}(y, x)=x \delta \omega(y, x),
\end{array}
$$

so that the $q_{i}$ 's vanish at the boundaries. This guarantees that the boundary conditions (3.6), (3.7), (3.8) and (3.9) are correctly imposed.

In the limit $\ell \rightarrow \infty$, our equations and results reduce to those of the asymptotically flat case studied in $[20,21]$. In this case, we explicitly proved that the ultraspinning zero-modes that we find cannot be pure gauge modes. That is, there is no pure gauge perturbation, obeying the boundary conditions we impose, which could potentially generate the regular metric perturbations that we consider. By continuity, we expect this property to hold true when the cosmological constant is switched on.

\section{Results and discussion}

The problem of finding the onset of the axisymmetric ultraspinning instability in the singlyspinning MP-AdS black hole reduces, at this point, to solving the equations (3.10) for the metric perturbations (3.12) in the TT gauge and that obey Dirichlet boundary conditions. The outcome of this analysis are the dimensionless negative modes, $\lambda r_{m}^{2}$, of the modified 

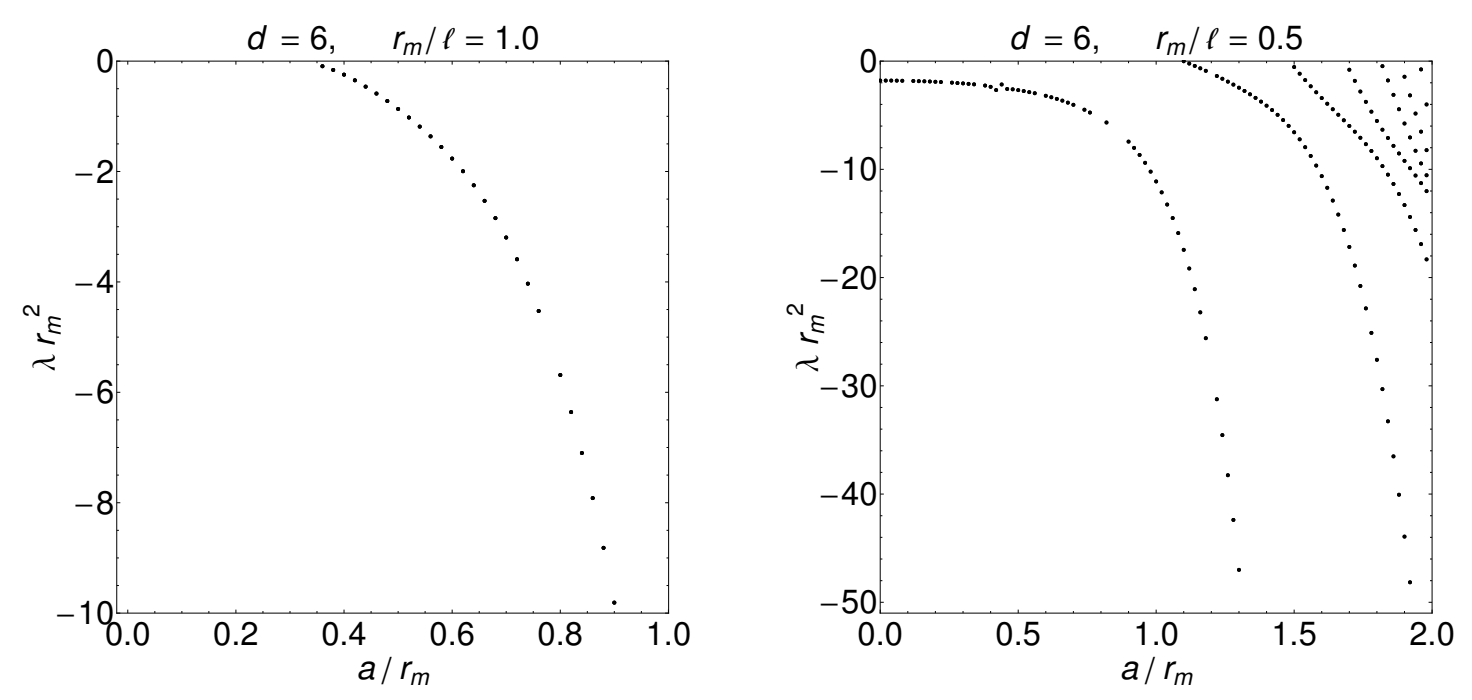

Figure 3. Dimensionless negative modes $\lambda r_{m}^{2}$ of the singly-spinning MP-AdS black hole in $d=6$ as a function of the dimensionless rotation parameter $a / r_{m}$ for fixed $r_{m} / \ell$. In the left plot, we represent the spectrum for $r_{m} / \ell=1.0$, while the right plot describes the spectrum for $r_{m} / \ell=0.5$. The latter has several (supposedly infinite) branches of zero-modes (for which $\lambda=0$ ) and the corresponding negative eigenvalues are labeled by the integer $l$. For this particular black hole family with $r_{m} / \ell=0.5$, the values of $a / r_{m}$ at which the first few branches intersect the $\lambda=0$ axis are: $a /\left.r_{m}\right|_{l=1} \simeq 1.10, a /\left.r_{m}\right|_{l=2} \simeq 1.49, a /\left.r_{m}\right|_{l=3} \simeq 1.69, a /\left.r_{m}\right|_{l=4} \simeq 1.81$, and $a /\left.r_{m}\right|_{l=5} \simeq 1.88$. As opposed to the thermodynamic $l=0,1$ zero-modes (curve on left plot, first two curves on right plot), the zero-modes with $l \geq 2$ describe the onset of ultraspinning instabilities of the black hole.

Lichnerowicz operator $\tilde{\Delta}_{L}$. These are obtained for each pair of parameters that specify the black hole for a fixed AdS radius $\ell$, namely the dimensionless mass radius, $r_{m} / \ell$, and the dimensionless rotation parameter, $a / r_{m}$. We will later describe the results in terms of the dimensionless asymptotic charges $M / \ell^{d-3}$ and $J / \ell^{d-2}$, which are more physical quantities. However, $r_{m}$ and $a$, which appear in the metric (2.1), are more convenient for the numerical analysis.

Examples of the spectrum of negative modes in $d=6$, as a function of the dimensionless rotation parameter, for two particular values of $r_{m} / \ell$, are presented in figure 3 . We expect that for higher $d$ the spectrum is qualitatively similar to $d=6$ (we explicitly confirmed this for $d=7)$.

The first property to notice is encoded in the curve on the left plot and on its counterpart on the right plot, which is the first curve counting from the left. This curve describes what we called the thermodynamic $l=0$ negative mode in subsection 2.2. As discussed there, it was proven in ref. [22] that this negative mode is always present if the black hole is asymptotically flat. However, the AdS curvature radius introduces a new scale in the system that changes this property. For black holes with small $r_{m} / \ell$ (namely for $r_{m} / \ell \lesssim 0.906$ in $d=6$ ) this negative mode is present for any value of $a / r_{m}$, see e.g. $r_{m} / \ell=0.5$ in the right plot of figure 3 , while for black holes with large $r_{m} / \ell$ (namely for $r_{m} / \ell \gtrsim 1.587$ in $d=6$ ) the thermodynamic $l=0$ eigenvalue is positive, independently of the rotation. 
When we fix $r_{m} / \ell$ to be in between these two regimes, the thermodynamic $l=0$ eigenvalue is positive for small $a / r_{m}$ and becomes negative for large $a / r_{m}$, see e.g. the $r_{m} / \ell=1.0$ case in the left plot of figure 3. The phase transition, where the thermodynamic $l=0$ eigenvalue is a zero-mode, occurs precisely at the critical rotation identified in (2.7), namely at $a /\left.r_{m}\right|_{l=0} \simeq 0.337$ (for $r_{m} / \ell=1.0$ and in $d=6$ ). This is what we expect from the thermodynamics discussion of subsection 2.2: in regions $B, C$ of figure 2 the thermodynamic $l=0$ eigenvalue is negative while in region $A$ it is positive. The agreement between the numerical and thermodynamic results is an important check of the numerical code.

The next interesting property of the spectrum of negative modes is encoded in the second curve counting from the left on the right plot of figure 3. According to figure 2, black holes with $r_{m} / \ell \lesssim 0.864$ in $d=6$ have a negative (positive) thermodynamic $l=1$ eigenvalue if the rotation parameter is such that the black hole is in region $C$ (region $B$ ), with the $l=1$ zero-mode curve defined by (2.8). The black hole family displayed on the right plot of figure 3 obeys these conditions, since $r_{m} / \ell=0.5$. Indeed, for rotations below (above) the critical rotation $a /\left.r_{m}\right|_{l=1} \simeq 1.10$, the thermodynamic $l=1$ eigenvalue is positive (negative) as predicted by (2.8).

The $l=0$ and $l=1$ negative eigenvalue curves are the only ones whose existence can be predicted by the analytical thermodynamic analysis of subsection 2.2. As discussed there, refs. $[22,29,40]$ proved that a zero-mode of the thermodynamic Hessian (2.6) is also a zero-mode of the (modified) Lichnerowicz operator (3.5), but the latter can have additional zero-modes, unrelated to that Hessian. The thermodynamic instabilities associated with the $l=0,1$ negative modes do not correspond to classical gravitational instabilities of the black hole. In particular, the thermodynamic $l=0,1$ zero-modes do not describe the onset of a classical instability. Instead, they describe gravitational perturbations that change the mass and the angular momentum of the black hole, within the MP-AdS family, but preserve the temperature and the angular velocity, due to the boundary conditions.

The thermodynamic $l=1$ zero-mode curve defines the ultraspinning surface of the system. It was conjectured in ref. [20] that only black holes which are outside this surface (i.e. in region $C$ of figure 2) might develop new zero/negative modes that have no thermodynamic interpretation, and that describe classical ultraspinning instabilities which are axisymmetric. The right plot of figure 3 confirms that the spectrum of MP-AdS black holes indeed has (a supposedly infinite sequence of) new branches of non-thermodynamic zeros-modes, for rotations strictly higher than the critical rotation $a /\left.r_{m}\right|_{l=1}$ associated with the $l=1$ zero-mode. These families exhibit an underlying harmonic structure (although the equations that we solve do not seem to separate into radial and angular equations). We use this property to suggestively label the several new branches by successive integers $l=2,3,4, \ldots$. This is an appropriate notation since the integer $l$ coincides with the number of nodes that the metric perturbations have on the horizon $y=0 .{ }^{8}$ The values of $a / r_{m}$ at which the first few branches intersect $\lambda=0$ for the particular family of black holes with $r_{m} / \ell=0.5$ in $d=6$ are summarised in the caption of figure 3 . The spectrum of black hole

\footnotetext{
${ }^{8}$ For the particular case where $\ell \rightarrow \infty$, this statement is illustrated in figure 3 of [21], where we plot the functions $\delta \chi(x, y)$ and $\delta \omega(x, y)$ for $l=1$ and $l=2$. The figures are qualitatively similar for finite $\ell$.
} 
families with $r_{m} / \ell \lesssim 0.906$ is qualitatively similar to this particular case.

In figure 4 , we plot the number of negative modes detected numerically, for a grid of points in the parameter plane $\left(r_{m} / \ell, a / r_{m}\right)$ in $d=6$ (left plot) and in $d=7$ (right plot). This figure completes the information displayed in figure 2 (in particular, for $d=6$, the black curves are the same plotted in figure 2). Not only it includes the numerical and analytical results for the thermodynamic $l=0,1$ modes but, in addition, it adds the numerical results relative to the $l \geq 2$ modes that have no thermodynamic interpretation. In region $A$ (see figure 2), we find no negative modes. In region $B$, the system has only the $l=0$ negative mode (blue dotted region in figure 4 ). In region $C$, the $l=1$ negative mode is also present, but additional negative modes appear for higher rotations. In figure 4, these are signaled by the red, purple, green, yellow and pink dotted areas. In the red area, only the $l=0,1$ modes are negative, and there is no classical instability. However, for higher rotation, in the purple area and above it, the $l=2$ harmonic becomes another negative mode of the modified Lichnerowicz operator (3.5). The transition from the red into the purple area defines the non-thermodynamic $l=2$ zero-mode curve. This surface signals the onset of the ultraspinning instability in the parameter space. The boundary of the purple and green areas identifies the non-thermodynamic $l=3$ zero-mode curve: above it the $l=3$ harmonic is also excited, i.e. it becomes a negative mode. Similarly, the green/yellow (yellow/pink) transition marks the non-thermodynamic $l=4(l=5)$ zeromode curve above which the $l=4(l=5)$ harmonic is also a negative mode. Not shown in this figure, there should be an infinite sequence of new excited harmonics. These would become visible if we considered higher values of $a / r_{m}$ in region $\mathrm{C}$.

In figure 5, we proceed to a thorough analysis of the singly-spinning MP-AdS parameter space. We represent the data in figure 4 for $d=6$ in terms of the dimensionless asymptotic conserved charges, $M \ell^{d-3}$ and $J \ell^{d-2}$, instead of using $r_{m}$ and $a$ to specify the black hole.

We do this for the sake of clarity, because the charges are more physical, and also because the map $\left(r_{m}, a\right) \rightarrow(M, J)$ in eqs. (2.2) is singular as $|a| \rightarrow \ell$, so that the parameter space looks very different. The physical parameter range is strictly below the curve $|J|=$ $M \ell$. To compare this figure with figure 4-left, notice that: (i) the asymptotically flat limit, which was the $r_{m} / \ell=0$ line in figure 4-left, is the point $(0,0)$ in figure 5; (ii) the line $a=\ell$, for finite $r_{m} / \ell$, in figure 4-left is now the asymptotic corner given by the limit $R \rightarrow \infty$ of $(R, R)$ in figure 5 , so that the various coloured regions do not all meet in the parameter space as suggested in figure 4-left; and (iii) the limit $a / r_{m} \rightarrow \infty$, within $a<\ell$, in figure 4-left is the curve $J=M \ell$ that puts a bound on the parameter space in figure 5 . Notice that, for relatively small fixed values of the mass (in AdS units), say $M / \ell^{d-3}=1$, there is always an $l=0$ negative mode, as in the asymptotically flat limit. On the other hand, there is a $l=0$ zero-mode for larger masses, say $M / \ell^{d-3}=2$. For any fixed value of the mass, we find that a sufficiently high rotation leads to the ultraspinning instabilities, in correspondence with figure 1.

Our study is limited to stationary perturbations. Therefore, we have not proven that our $l \geq 2$ negative modes do indeed correspond to a region in parameter space where some black hole perturbations grow exponentially with time. Including time-dependence is not conceptually difficult but it is only computationally harder. Additional metric components 

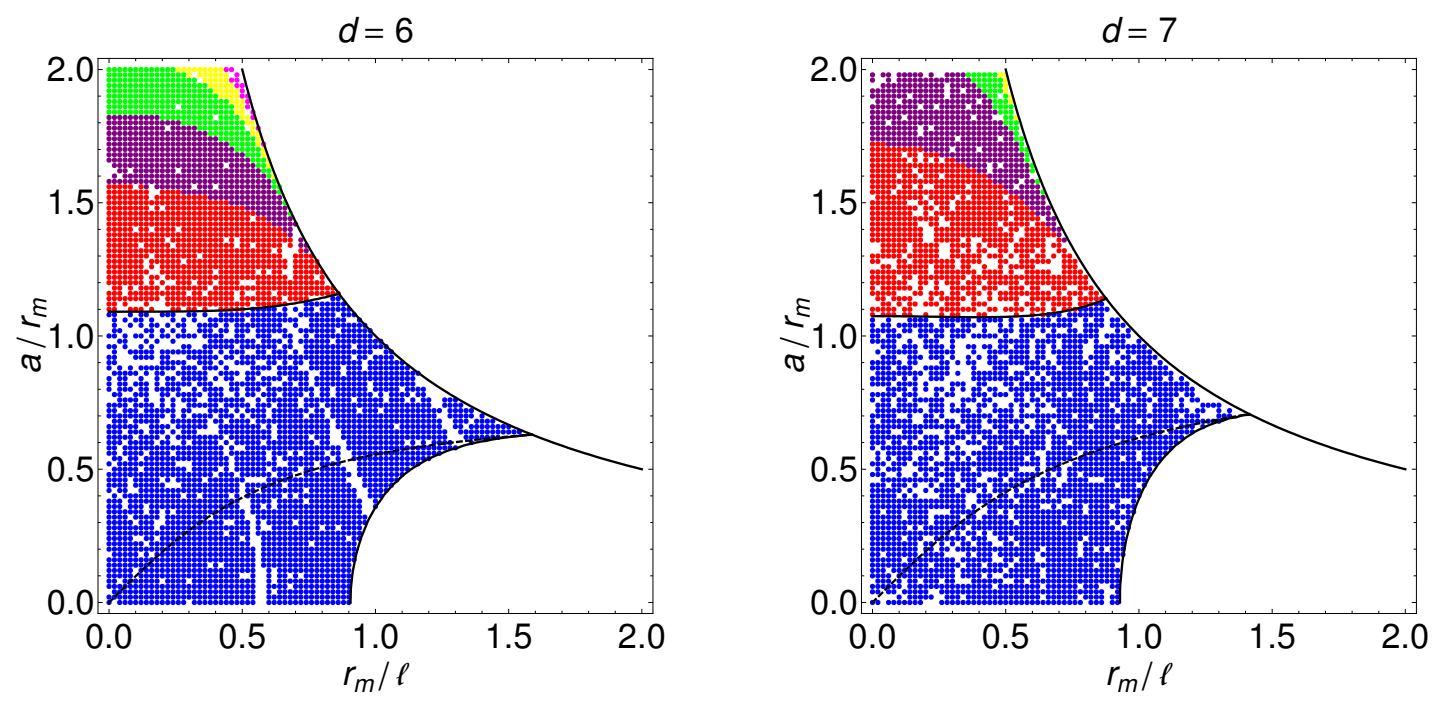

Figure 4. Number of negative modes of the singly-spinning MP-AdS black hole in $d=6$ (left) and $d=7$ (right). The plots describe the dimensionless rotation parameter $a / r_{m}$ as a function of the dimensionless mass-radius $r_{m} / \ell$. As we move from the bottom to the top, the new colored/dotted areas represent regions where a new negative mode of the (modified) Lichnerowicz operator gets excited: blue (one negative mode), red (2 n.m.), purple (3 n.m.), green (4 n.m.), yellow (5 n.m.), pink (6 n.m.), ... The interpretation of the several curves plotted is described in figure 2. (Note that the faults in these figures, e.g. where we expected to find blue dots, correspond to parameter space points where our numerical code failed. They do not correspond to black holes with no negative modes.)

would have to be perturbed (e.g. $h_{t r}, \ldots$ ) in (3.1), and we would have to solve numerically a system of many more PDEs. This problem is however of fundamental interest since its solution would provide a definite proof of the ultraspinning instability in MP-AdS black holes, together with information on the instability timescale. Having said this, we nevertheless claim that we have found the stationary zero-modes that signal the onset of the ultraspinning instability. Our confidence comes from the fact that the analogous stability problem, including time-dependence, was studied in [22] for asymptotically flat cohomogeneity-1 MP black holes (equal angular momenta, odd $d$ ). There, the analogues of the $l=1,2,3,4, \ldots$ zero-modes are also present, and the corresponding perturbation sectors decouple. The time-dependent analysis confirmed the absence of a classical black hole instability in the $l=1$ sector of perturbations, and the existence of an ultraspinning instability in the $l \geq 2$ sectors. We take this result as good evidence in support of a similar interpretation in the singly-spinning MP-AdS case.

The ultraspinning $l \geq 2$ zero-modes do not admit a thermodynamic interpretation, as we have stressed. They should correspond not only to the onset of classical instabilities, but also to the bifurcation to new stationary AdS black hole families with 'pinched' horizons, which have the same isometries as the MP-AdS black holes. This is the conjecture of ref. [25] (following $[12,16]$ in the asymptotically flat case), represented in figure 1. In particular, the $l=2$ zero-mode should connect to a family of AdS black holes which are 'pinched' on 


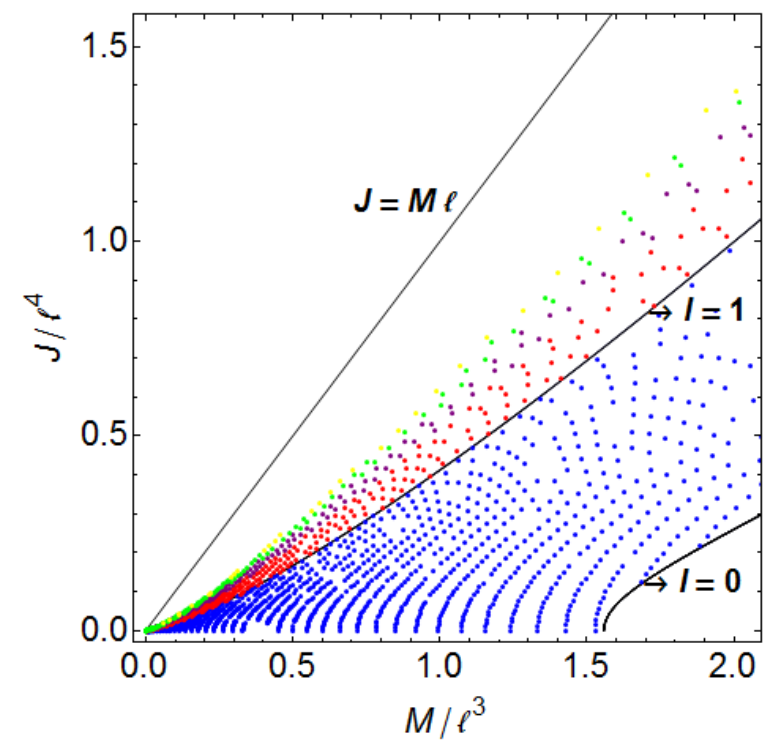

Figure 5. Number of negative modes of the singly-spinning MP-AdS black hole in $d=6$. This figure has the data of figure 4-left, now plotting the dimensionless angular momentum $J / \ell^{d-2}$ as a function of the dimensionless mass $M / \ell^{d-3}$. Regular MP-AdS black holes exist for $|J|<M \ell$. We represent the location of the $l=0$ and the $l=1$ zero-modes, for reference.

the poles. The location of the $l=2$ zero-mode is represented as point $I$ in figure 1 . At some point along the phase diagram of this new family, a horizon topology phase transition should connect it to the AdS black ring with the same isometries, which has been constructed perturbatively in the limit of large dimensionless angular momentum [25]. Similarly, the $l=3(l=4)$ zero-mode, identified as point $I I(I I I)$ in figure 1 , should connect to a new family that interpolates between the MP-AdS family and the AdS black Saturn (concentric rings), constructed perturbatively in [25]. Our results support the conjecture of ref. [25] not only because we find the bifurcation points, but also because the shape of the numerical perturbations, with its harmonic structure labelled by $l$, is consistent with the appropriate 'pinches' of the horizon (footnote 8).

As discussed in subsection 2.3, all the ultraspinning unstable MP-AdS black holes in the purple, green, yellow, pink, .. dotted areas of figure 4 have $\Omega_{H} \ell>1$, and thus all of them are also afflicted by the superradiant instability [31-33]. The instability is expected to be inherited by the 'pinched' black hole families, at least close to the bifurcation point.

An interesting open problem is what is the interpretation of the ultraspinning instability and of the associated new AdS pinched black hole phases in the holographic dual field theory. Steps in this direction have already been taken in the context of the Scherk-Schwarz compactified AdS. In this case, in the hydrodynamic limit of the holographic theory one finds: i) pinched plasma balls $[49,50]$ that should be in correspondence with the gravitational ultraspinning system, ii) deformed plasma tubes [51-54] that should be dual to the Gregory-Laflamme instability of Scherk-Schwarz-AdS black strings and iii) rotating plasma ball instabilities $[55,56]$ that should describe the gravitational bar-mode instability $[34,35]$ mentioned in the Introduction. 


\section{Acknowledgments}

It is a pleasure to acknowledge the stimulating discussions with our collaborators Roberto Emparan and Harvey Reall in the ultraspinning project. OJCD acknowledges financial support provided by the European Community through the Intra-European Marie Curie contract PIEF-GA-2008-220197. This work was partially funded by FCT-Portugal through projects PTDC/FIS/099293/2008, CERN/FP/83508/2008 and CERN/FP/109306/2009. $\mathrm{PF}$ was a member of the CPT group at the Department of Mathematical Sciences, Durham University, and was supported by an STFC rolling grant while part of this work was carried out. PF is supported by an EPSRC postdoctoral fellowship [EP/H027106/1].

Open Access. This article is distributed under the terms of the Creative Commons Attribution Noncommercial License which permits any noncommercial use, distribution, and reproduction in any medium, provided the original author(s) and source are credited.

\section{References}

[1] R. Emparan and H.S. Reall, Black Holes in Higher Dimensions, Living Rev. Rel. 11 (2008) 6 [arXiv:0801.3471] [SPIRES].

[2] R. Emparan and H.S. Reall, A rotating black ring in five dimensions, Phys. Rev. Lett. 88 (2002) 101101 [hep-th/0110260] [SPIRES].

[3] R.C. Myers and M.J. Perry, Black Holes in Higher Dimensional Space-Times, Ann. Phys. 172 (1986) 304 [SPIRES].

[4] H. Elvang and P. Figueras, Black Saturn, JHEP 05 (2007) 050 [hep-th/0701035] [SPIRES].

[5] H. Iguchi and T. Mishima, Black di-ring and infinite nonuniqueness, Phys. Rev. D 75 (2007) 064018 [hep-th/0701043] [SPIRES].

[6] J. Evslin and C. Krishnan, The Black Di-Ring: An Inverse Scattering Construction, Class. Quant. Grav. 26 (2009) 125018 [arXiv:0706.1231] [SPIRES].

[7] K. Izumi, Orthogonal black di-ring solution, Prog. Theor. Phys. 119 (2008) 757 [arXiv: 0712.0902] [SPIRES].

[8] H. Elvang and M.J. Rodriguez, Bicycling Black Rings, JHEP 04 (2008) 045 [arXiv:0712.2425] [SPIRES].

[9] H. Elvang, R. Emparan and P. Figueras, Phases of Five-Dimensional Black Holes, JHEP 05 (2007) 056 [hep-th/0702111] [SPIRES].

[10] R. Emparan and P. Figueras, Multi-black rings and the phase diagram of higher-dimensional black holes, JHEP 11 (2010) 022 [arXiv: 1008.3243] [SPIRES].

[11] H. Iguchi and T. Mishima, Thermodynamic black di-rings, Phys. Rev. D 82 (2010) 084009 [arXiv: 1008.4290] [SPIRES].

[12] R. Emparan, T. Harmark, V. Niarchos, N.A. Obers and M.J. Rodriguez, The Phase Structure of Higher-Dimensional Black Rings and Black Holes, JHEP 10 (2007) 110 [arXiv: 0708.2181] [SPIRES].

[13] R. Emparan, T. Harmark, V. Niarchos and N.A. Obers, Blackfolds, Phys. Rev. Lett. 102 (2009) 191301 [arXiv:0902. 0427] [SPIRES]. 
[14] R. Emparan, T. Harmark, V. Niarchos and N.A. Obers, Essentials of Blackfold Dynamics, JHEP 03 (2010) 063 [arXiv:0910.1601] [SPIRES].

[15] R. Emparan, T. Harmark, V. Niarchos and N.A. Obers, New Horizons for Black Holes and Branes, JHEP 04 (2010) 046 [arXiv: 0912.2352] [SPIRES].

[16] R. Emparan and R.C. Myers, Instability of ultra-spinning black holes, JHEP 09 (2003) 025 [hep-th/0308056] [SPIRES].

[17] R. Gregory and R. Laflamme, Black strings and p-branes are unstable, Phys. Rev. Lett. 70 (1993) 2837 [hep-th/9301052] [SPIRES].

[18] S.S. Gubser, On non-uniform black branes, Class. Quant. Grav. 19 (2002) 4825 [hep-th/0110193] [SPIRES].

[19] T. Wiseman, Static axisymmetric vacuum solutions and non-uniform black strings, Class. Quant. Grav. 20 (2003) 1137 [hep-th/0209051] [SPIRES].

[20] O.J.C. Dias, P. Figueras, R. Monteiro, J.E. Santos and R. Emparan, Instability and new phases of higher-dimensional rotating black holes, Phys. Rev. D 80 (2009) 111701 [arXiv: 0907.2248] [SPIRES].

[21] O.J.C. Dias, P. Figueras, R. Monteiro and J.E. Santos, Ultraspinning instability of rotating black holes, Phys. Rev. D 82 (2010) 104025 [arXiv: 1006.1904] [SPIRES].

[22] O.J.C. Dias, P. Figueras, R. Monteiro, H.S. Reall and J.E. Santos, An instability of higher-dimensional rotating black holes, JHEP 05 (2010) 076 [arXiv:1001.4527] [SPIRES].

[23] J.M. Maldacena, The large-N limit of superconformal field theories and supergravity, Int. J. Theor. Phys. 38 (1999) 1113 [Adv. Theor. Math. Phys. 2 (1998) 231] [hep-th/9711200] [SPIRES].

[24] O. Aharony, S.S. Gubser, J.M. Maldacena, H. Ooguri and Y. Oz, Large-N field theories, string theory and gravity, Phys. Rept. 323 (2000) 183 [hep-th/9905111] [SPIRES].

[25] M.M. Caldarelli, R. Emparan and M.J. Rodriguez, Black Rings in (Anti)-deSitter space, JHEP 11 (2008) 011 [arXiv:0806.1954] [SPIRES].

[26] J. Armas and N. A. Obers, to appear.

[27] S.W. Hawking, C.J. Hunter and M. Taylor, Rotation and the AdS/CFT correspondence, Phys. Rev. D 59 (1999) 064005 [hep-th/9811056] [SPIRES].

[28] P.T. Chrusciel, D. Maerten and P. Tod, Rigid upper bounds for the angular momentum and centre of mass of non-singular asymptotically anti-de Sitter space-times, JHEP 11 (2006) 084 [gr-qc/0606064] [SPIRES].

[29] R. Monteiro, M.J. Perry and J.E. Santos, Semiclassical instabilities of Kerr-AdS black holes, Phys. Rev. D 81 (2010) 024001 [arXiv: 0905. 2334] [SPIRES].

[30] T. Prestidge, Dynamic and thermodynamic stability and negative modes in Schwarzschild-anti-de Sitter, Phys. Rev. D 61 (2000) 084002 [hep-th/9907163] [SPIRES].

[31] V. Cardoso and O.J.C. Dias, Small Kerr-anti-de Sitter black holes are unstable, Phys. Rev. D 70 (2004) 084011 [hep-th/0405006] [SPIRES].

[32] H.K. Kunduri, J. Lucietti and H.S. Reall, Gravitational perturbations of higher dimensional rotating black holes: Tensor Perturbations, Phys. Rev. D 74 (2006) 084021 [hep-th/0606076] [SPIRES]. 
[33] V. Cardoso, O.J.C. Dias and S. Yoshida, Classical instability of Kerr-AdS black holes and the issue of final state, Phys. Rev. D 74 (2006) 044008 [hep-th/0607162] [SPIRES].

[34] M. Shibata and H. Yoshino, Nonaxisymmetric instability of rapidly rotating black hole in five dimensions, Phys. Rev. D 81 (2010) 021501 [arXiv:0912.3606] [SPIRES].

[35] M. Shibata and H. Yoshino, Bar-mode instability of rapidly spinning black hole in higher dimensions: Numerical simulation in general relativity, Phys. Rev. D 81 (2010) 104035 [arXiv: 1004 .4970] [SPIRES].

[36] B. Carter, Hamilton-Jacobi and Schrödinger separable solutions of Einstein's equations, Commun. Math. Phys. 10 (1968) 280 [SPIRES].

[37] G.W. Gibbons, M.J. Perry and C.N. Pope, The First Law of Thermodynamics for Kerr-Anti-de Sitter Black Holes, Class. Quant. Grav. 22 (2005) 1503 [hep-th/0408217] [SPIRES].

[38] M.M. Caldarelli, G. Cognola and D. Klemm, Thermodynamics of Kerr-Newman-AdS black holes and conformal field theories, Class. Quant. Grav. 17 (2000) 399 [hep-th/9908022] [SPIRES].

[39] S.W. Hawking and D.N. Page, Thermodynamics of Black Holes in anti-de Sitter Space, Commun. Math. Phys. 87 (1983) 577 [SPIRES].

[40] H.S. Reall, Classical and thermodynamic stability of black branes, Phys. Rev. D 64 (2001) 044005 [hep-th/0104071] [SPIRES].

[41] R. Monteiro, M.J. Perry and J.E. Santos, Thermodynamic instability of rotating black holes, Phys. Rev. D 80 (2009) 024041 [arXiv: 0903.3256] [SPIRES].

[42] S.S. Gubser and I. Mitra, Instability of charged black holes in anti-de Sitter space, hep-th/0009126 [SPIRES].

[43] S.S. Gubser and I. Mitra, The evolution of unstable black holes in anti-de Sitter space, JHEP 08 (2001) 018 [hep-th/0011127] [SPIRES].

[44] D.J. Gross, M.J. Perry and L.G. Yaffe, Instability of Flat Space at Finite Temperature, Phys. Rev. D 25 (1982) 330 [SPIRES].

[45] O.J.C. Dias, R. Monteiro, H.S. Reall and J.E. Santos, A scalar field condensation instability of rotating anti-de Sitter black holes, JHEP 11 (2010) 036 [arXiv:1007.3745] [SPIRES].

[46] I.Y. Park, C.N. Pope and A. Sadrzadeh, AdS braneworld Kaluza-Klein reduction, Class. Quant. Grav. 19 (2002) 6237 [hep-th/0110238] [SPIRES].

[47] R. Gregory, Black string instabilities in anti-de Sitter space, Class. Quant. Grav. 17 (2000) L125 [hep-th/0004101] [SPIRES].

[48] L.N. Trefethen, Spectral Methods in MATLAB, SIAM, Philadelphia (2000).

[49] S. Lahiri and S. Minwalla, Plasmarings as dual black rings, JHEP 05 (2008) 001 [arXiv: 0705.3404] [SPIRES].

[50] S. Bhardwaj and J. Bhattacharya, Thermodynamics of Plasmaballs and Plasmarings in $3+1$ Dimensions, JHEP 03 (2009) 101 [arXiv:0806.1897] [SPIRES].

[51] V. Cardoso and O.J.C. Dias, Gregory-Laflamme and Rayleigh-Plateau instabilities, Phys. Rev. Lett. 96 (2006) 181601 [hep-th/0602017] [SPIRES]. 
[52] V. Cardoso, O.J.C. Dias and L. Gualtieri, The return of the membrane paradigm? Black holes and strings in the water tap, Int. J. Mod. Phys. D 17 (2008) 505 [arXiv:0705.2777] [SPIRES].

[53] M.M. Caldarelli, O.J.C. Dias, R. Emparan and D. Klemm, Black Holes as Lumps of Fluid, JHEP 04 (2009) 024 [arXiv:0811.2381] [SPIRES].

[54] M.M. Caldarelli, O.J.C. Dias and D. Klemm, Dyonic AdS black holes from magnetohydrodynamics, JHEP 03 (2009) 025 [arXiv:0812.0801] [SPIRES].

[55] V. Cardoso and O.J.C. Dias, Bifurcation of Plasma Balls and Black Holes to Lobed Configurations, JHEP 04 (2009) 125 [arXiv: 0902.3560] [SPIRES].

[56] V. Cardoso, O.J.C. Dias and J.V. Rocha, Phase diagram for non-axisymmetric plasma balls, JHEP 01 (2010) 021 [arXiv:0910.0020] [SPIRES].

[57] Y. Brihaye, T. Delsate and E. Radu, On the stability of AdS black strings, Phys. Lett. B 662 (2008) 264 [arXiv: 0710.4034] [SPIRES]. 